\title{
NO COMMON GENE EXPRESSION ALTERATIONS BASED ON MUCOSITIS SEVERITY IN THREE INDEPENDENT CANCER COHORTS OF ACUTE MYELOID LEUKAEMIA, MULTIPLE MYELOMA AND TONSIL SQUAMOUS CELL CANCER
}

\author{
Mette Marcussen ${ }^{1,2}$, Mads Sønderkær ${ }^{2}$, Julie Støve Bødker², Ilse Christiansen³, Olav J. Bergmann ${ }^{4}$, Martin Bøgsted ${ }^{1,2,3}$, \\ Karen Dybkær ${ }^{1,2,3}$, Hans Erik Johnsen ${ }^{1,2,3}$
}

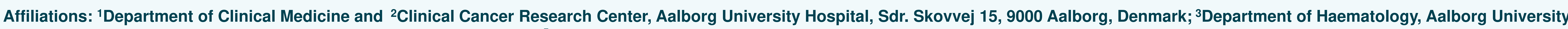
Hospital, Hobrovej 18-22, 9000 Aalborg, Denmark; ${ }^{5}$ School of Dentistry, Faculty of Health Science, Aarhus University; Vennelyst Boulevard 9, 8000 Aarhus, Denmark

Introduction: Mucositis is a frequent clinical complication among patients receiving cancer therapy. However, the molecular mechanisms are not fully understood'. Objectives: Our aim was to identify common mucositis and disease related global gene expression (GGE) profiles among patients in three independent cancer treatment cohorts: acute myeloid leukemia (AML), multiple myeloma (MM), and tonsil squamous cell carcinoma (TSCC). Data from the latter two was previously published ${ }^{2,3}$. Methods: Three consecutive buccal biopsies from six de novo AML patients, ten patients with MM, eight patients with TSCC and nine healthy, age and gender matched individuals (CON) had GGE data extracted using the Affymetrix GeneChip Human Exon 1.0 Array. Statistical analyses were performed using $R$ version 3.4.2 and Bioconductor packages. Clinical mucositis was correlated to GGE.
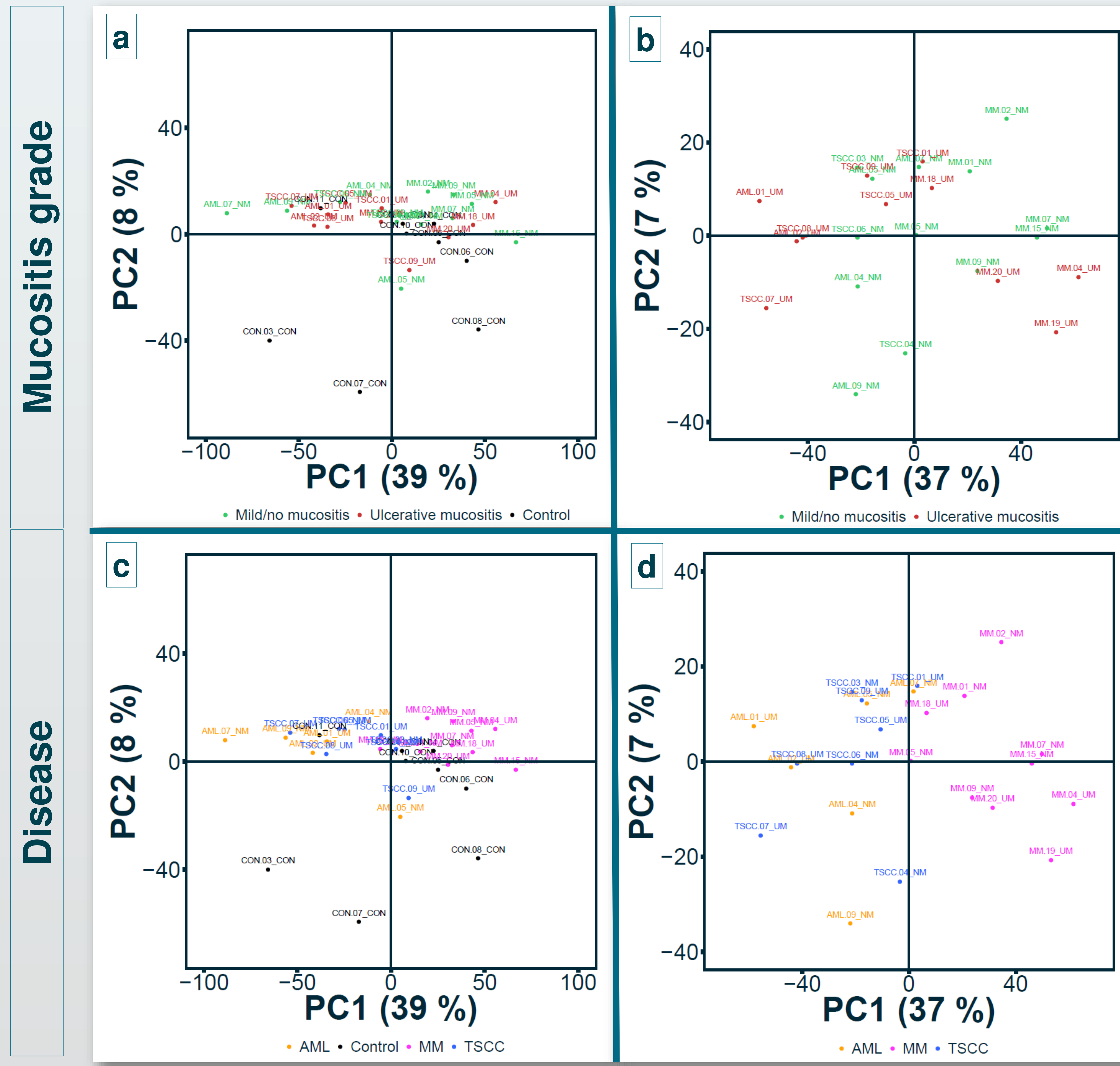

Before treatment

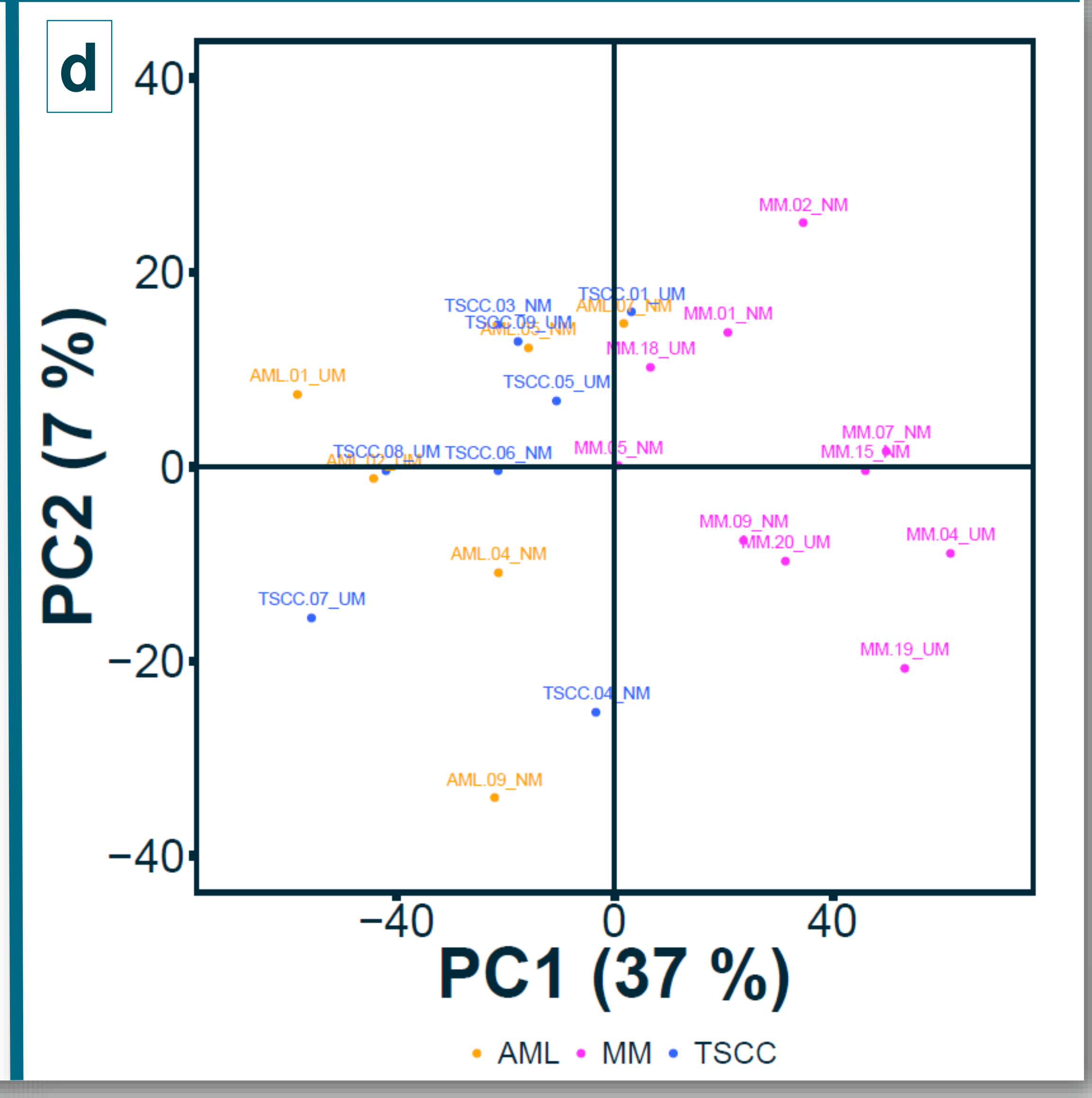

Results A principal component analysis (PCA) combining three independent cancer cohorts, AML, MM and TSCC did not identify common GGE patterns based on clinical mucositis severity, se Figure 1, a and b. However, we saw a tendency to cluster according to disease, see Figure 1, c and d. Previously published GGE data on the MM and TSCC cohort identified dissimilar potential biomarkers for mucositis severity, both related to immune response.

Conclusions The mucosal GGE profiles from three independent cancer cohorts did not identify common GGE alterations based on the severity of clinical mucositis.

Figure 1. PCA-plots of mucosal global gene expression from three independent cancer treatment cohorts before ( $a$ and $c$ ) and during (b and d) cancer treatment. PCA plot are colored by mucositis grade ( $a$ and $b$ ) and disease ( $c$ and $d)$.

Abbreviations: Green=no/mild mucositis (NM); Red=ulcerative mucositis (UM); Pink=patients with multiple myeloma (MM); Blue=patients with tonsillar squamous cell cancer (TSCC); Yellow=patients with acute lymphoid leukaemia (AML) Black=healthy age and gender matched individuals

1. Marcussen M, et al. A systematic review of molecular responses to cancer therapy in normal human mucosa. TripleO: https://doi.org/10.1016/i.0000.2017.08.002

2. Marcussen M, et al. Molecular Characteristics of High-Dose Melphalan Associated Oral Mucositis in Patients with Multiple Myeloma : A Gene Expression Study on Human Mucosa. PlosOne 2017: http://dx.doi.org/10.1371/journal.pone.0169286

3. Marcussen M et al. Oral mucosa tissue gene expression profiling before, during, and after radiation therapy for tonsil squamous cell carcinoma. PlosOne 2018 http://dx.doi.org/10.1371/journal.pone.0190709
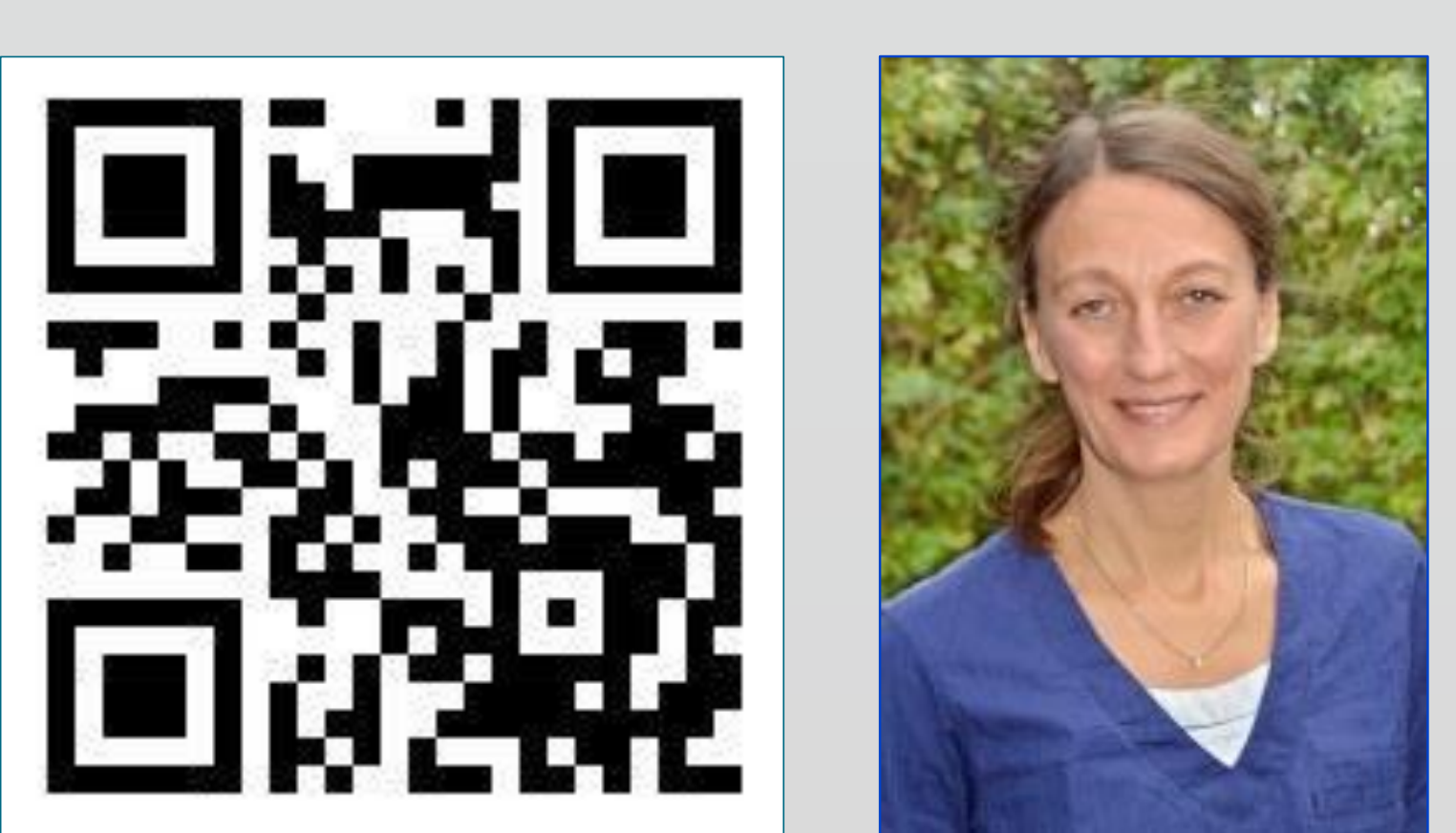